\title{
Vibration Analysis for Monitoring of Ancient Tie-Rods
}

\author{
L. Collini, R. Garziera, and K. Riabova \\ Department of Engineering and Architecture, University of Parma, Viale Delle Scienze 181/A, 43124 Parma, Italy \\ Correspondence should be addressed to K. Riabova; ksenia.riabova@gmail.com
}

Received 26 April 2017; Accepted 20 June 2017; Published 20 August 2017

Academic Editor: Pedro Galvín

Copyright (C) 2017 L. Collini et al. This is an open access article distributed under the Creative Commons Attribution License, which permits unrestricted use, distribution, and reproduction in any medium, provided the original work is properly cited.

\begin{abstract}
This paper presents an application of vibration analysis to the monitoring of tie-rods. An algorithm for the axial load estimation based on experimentally measured natural frequencies is introduced and its application to a case study is reported. The proposed model of a tie-rod incorporates elastic bed-type boundary conditions that represent the contact between stonework and the tierod. The weighed differences between experimentally and numerically determined frequencies are minimized with respect to the parameters of the model, the main being the axial load and the stiffness at the tie-rod/wall interface. Thus, the multidimensional optimization problem is solved. Results are analysed in comparison to a model with simple fixed-end boundary conditions. In addition, the analytical formulation of the problem is delivered.
\end{abstract}

\section{Introduction}

The present paper reviews the applications of vibration analysis to the monitoring of the so-called "tie-rods." Tie-rods are metal beams used in a wide range of civil constructions. The main purpose of these structural elements is to provide support for masonry arches and vaults in ancient buildings, like churches, cathedrals, and castles, which are known to lurch and founder in course of time. Tie-rods are subjected to axial tension and, thus, help the building resist lateral loads exerted by walls and facades. Figure 1 pictures a typical layout of tie-rods supporting arches of the first floor terrace in the medieval Castello di Torrechiara (Castle of Torrechiara) in the province of Parma, Italy.

Over the years, deformations of masonry walls and eventual displacements in the building may cause significant changes in the axial loads of tie-rods. In the extremes, this can lead to either of two scenarios: failure in structural integrity of tie-rods (damages and cracks) or loss of loads and subsequent performance decline, a phenomenon referred to as the "laziness" of tie-rods. Both of the scenarios are dangerous for the safety and integrity of buildings and can lead to irretrievable harm to the precious historical heritage of the human race. For this reason regular monitoring of tierods' condition is of a great importance.

Health monitoring of tie-rods includes two major steps. The first one is identification of axial load and the second one is damage identification. As for the first one, multiple methods have been developed to accomplish this task and the details on the state of art are provided in the next section. Such experimental techniques should be as less invasive as possible and at the same time provide sufficient data on the beam condition. Generally this type of testing is referred to as "nondestructive."

In particular, nondestructive testing (NDT) is the process of investigating structures and elements for characteristics, discontinuities, changes in properties, and so forth without harming the continuity and usability of the part under testing. One of the relatively cheap, easily executable, and reliable NDT techniques is vibration analysis (VA). This way of health monitoring of the structures can be applied to testing whole buildings as well as its smallest parts depending on the scope and approach used. VA is based on investigation of dynamics of a structure under a certain excitation: it can be an impact hammer or a shaker. The response to the excitation is registered via sensors: accelerometers, optic sensors, laser, and so forth. Vibrational response contains information about the main structural characteristics of the system: mass and stiffness. Based on the knowledge of modal parameters, conclusions are drawn on the loading and structural integrity of the elements.

A reliable experimental technique helps to validate analytical and numerical models used for prediction of cracked beam dynamics. The purpose of this research is to develop a 


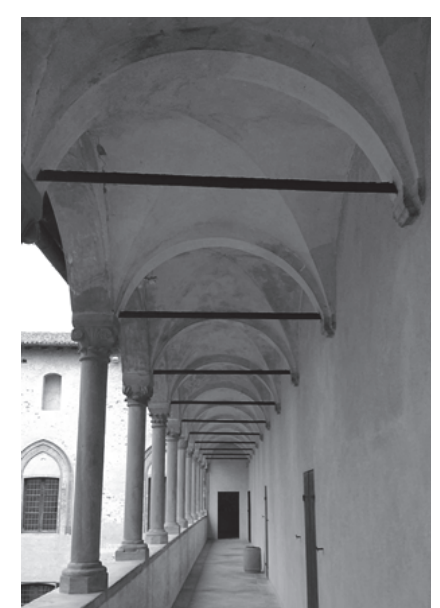

Figure 1: Tie-rods in the Castle of Torrechiara in Langhirano, Parma, Italy.

VA procedure based on quantitative and qualitative analysis of frequency response functions.

The further sections describe a method for axial load identification in tie-rods developed by the Department of Industrial Engineering of the University of Parma. This approach combines in situ dynamic tests and computations that make use of a beam model with complex boundary conditions. The method was tested and improved throughout some years since it was applied for multiple case studies of monitoring such famous Italian historic buildings as Duomo di Parma (Cathedral of Parma), Basilica della Madonna dell'Umiltà in Pistoia (Church of Our Lady of Humility), Rocca Sanvitale di Fontanellato (Sanvitale Fortress of Fontanellato), and Casa Romei (Romeì House) in Ferrara, as reported by the authors Collini et al. in [1-7].

\section{State of the Art in Axial Load Identification in Tie-Rods}

The structural characterization of tie-rods is crucial for the safety assessment of historical buildings. The main parameters that characterize the behaviour of tie-rods are the tensile force, the modulus of elasticity of the material, and the rotational stiffness at both restraints. In the last decades several techniques for an indirect nondestructive evaluation of such parameters have been proposed. The nondestructive procedures currently available for the structural characterization of tie-rods can be grouped in static, static-dynamic, and pure dynamic approaches. Pioneering static methods presented, for instance, in works of Pozzati [8] and in [9, $10]$, in spite of minor differences, are based on measures of displacement and/or strain at few cross-sections of the tierod due to applied static loads. Bati and Tonietti introduced a static approach for force identification that consisted of measuring three vertical displacements and strains variations at three sections of the tie-rod under a concentrated load [10]. Even if the data postprocessing is quite straightforward, these methods are extremely sensitive to the experimental error in the measures of displacement. In addition, since tie-rods are usually positioned at considerable heights, the need of measuring vertical deflections with respect to a reference fixed base makes static methods difficult in practice.

Mixed approaches try to identify the unknown parameters by combining static and dynamic measures. Blasi and Sorace [11-13] modelled tie-rods as simply supported Euler beams with rotational springs of similar stiffness added on each edge. The stiffness of the spring and the force were the two unknowns obtained from the system of equations, built with a static equation for deflection and a dynamic equation for natural frequencies. Thus, this method required data from two separate experiments, that is, in situ measurements of the central deflection under static load and of the fundamental natural frequency, which can be obtained by hammer impact testing and Fourier transforming the recorded accelerations. Testing of this method in laboratory conditions showed good results; however, measurement errors can cause significant deviation in results for the two unknowns. Even though static-dynamic methods can exploit additional dynamic information for the characterization, they are still affected by the shortcomings related to deflection measurements.

Such drawbacks are avoided in pure dynamic procedures [14-28], where, in general, the difference between the experimental and the calculated natural frequencies of vibration is minimized in order to identify the unknown parameters.

Lagomarsino and Calderini [14] developed an algorithm to identify the axial tensile force in ancient tie-rods by using the first three natural frequencies. The tie-rod was modelled as an Euler beam of uniform cross-section, neglecting the shear deformation and rotary inertia, and was assumed to be simply supported at the ends with additional rotational springs.

Recently Maes et al. introduced a method that enables definition of axial loads in slender beams with unknown boundary conditions, taking into account effects of rotational inertia of the beam and masses of sensors [15]. However, it requires data from five or more sensors along the length of the beam to determine all the introduced unknowns of the inverted problem. A similar technique of the axial force identification was developed by $\mathrm{Li}$ et al. [16], focusing on studies of Euler-Bernoulli beams and takes into account bending stiffness effects.

Rebecchi et al. established an analytical method of processing experimental data from five instrumented sections of a prismatic slender beam, which showed excellent results in estimation of the axial load in tie-rods [17]. The method does not require any exact value of effective length of the beam but neglects both rotary inertia and shear deformations effects in the solution for beam vibrations. For cases of similar beams their colleagues Tullini et al. proposed a static method of axial force identification [18-20]. The analytical algorithm makes use of any set of experimental data represented by flexural displacements or curvatures measured at five crosssections of the beam subjected to an additional concentrated lateral load. Gentilini et al. developed in [21] a procedure that combines dynamical testing with FEM simulations using added masses. The method was tested out for tierods of various lengths and load intensity, showing reliable results. 


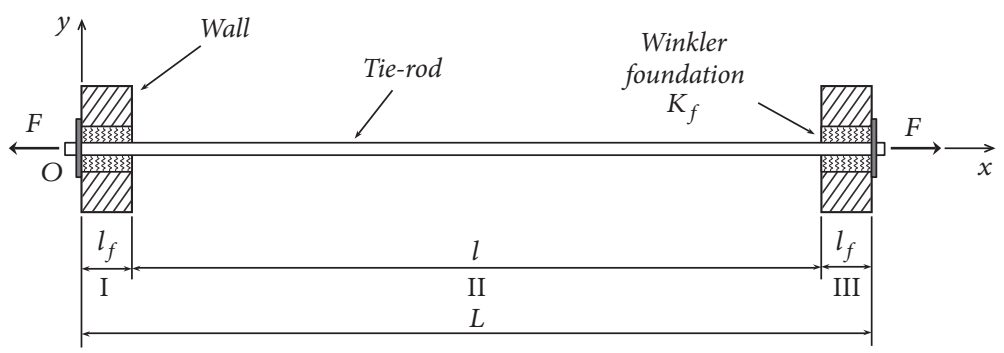

FIGURE 2: Tie-rod with elastic bed-type boundaries.

Livingston et al. identified the tensile force in prismatic beams of uniform section by using modal data and assuming rotational and vertical springs at each end of the beam [22]. Shear deformation and rotary inertia were neglected (according to the Euler beam model).

Another fully dynamic procedure has been proposed by Kim and Park [23]. It allows identifying the tension force and flexural and axial stiffness of the cable from measured natural frequencies. Anyway this technique is not immediately applicable to tie-rods since they cannot be modelled as cables and present uncertain constraints due to the portion of the rod inserted into the masonry wall or column.

Amabili et al. [1-6] developed a two-step method consisting of in situ measurements of tie-rods' natural frequencies and further elaboration of the data via an optimization algorithm based on the Rayleigh-Ritz method [7]. Varying certain parameters, the main of which was the sought axial load, this algorithm matched estimated sets of frequencies with those determined from experiments. The considered numerical models allowed analysis of ancient tie-rods affected by nonperfect constraints, added masses, discontinuities, irregular cross-sections, and complex boundary conditions. Different importance of the natural frequencies can be as well taken into account. The technique is of simple execution and allows minimizing the measurement error. Its functionality and reliability have been proved as it has been applied with success to many case studies.

\section{Dynamic Method for Load Identification}

The first step of the method is the in situ experimental identification of natural frequencies of the tie-rods by measuring the frequency response functions (FRFs) via instrumented hammer excitation. Precisely the testing technique used for the investigation of tie-rods in Casa Romei located in the city of Ferrara, Emilia-Romagna, Italy, was described in [1-3]. The first four to six natural frequencies, which can be extracted with high precision, were in the focus. Subsequently, an axially loaded tie-rod was modelled in finite element method (FEM) software Abaqus 6.13 as a general case of a Timoshenko beam, using three-dimensional beam elements retaining shear deformation and rotational inertia. The parametric model enabled considering nonuniform crosssections of rods, since this is often the case for hand-made tie-rods in old buildings.

The interface tie-rod/wall was assumed to be a continuous elastic bed; that is, extremities of tie-rods inserted inside masonry walls were modelled as resting on Winkler-type foundation. This type of boundary has been used in dynamics of particular cases, for example, for beams or rails subjected to travelling loads, as reported by Farghaly and Zeid [29], Ruge and Birk [30], and Koroma et al. [31]. In our case we discretized the elastic bed into separate springs equispaced along the length of the bed $l_{f}$, each of a stiffness $k_{f}$ (see Figure 2).

Clearly, the foundation may have a nonuniformly distributed stiffness, which would result in different $k_{f}$ assigned to each spring. The advantage of the Winkler bed compared to other types of boundaries generally used for tie-rods is that a number of springs placed closely exhibit more complex behaviour than linear and/or rotational springs attached to a single node. Hence, it is a reliable way of modelling a real wall-rod contact condition. The optimization parameters in this case were the unknown axial load, the stiffness of the foundation, and the length of the rod inside the wall. In real cases the lengths of the extremities inserted into the masonry and the stiffness of the foundation most likely differ for each end of the tie-rod. This complication has been avoided in the reported case study; however, the method proposed hereby is capable of taking into account other desired parameters of the model.

3.1. Analytical Formulation of the Problem. As shown in Figure 2, tie-rod was divided into three sections of length $l_{f}$, section I; $l$, section II; and again $l_{f}$, section III, where $l_{f}$ identifies the portion inserted into the wall and $l$ is the measured "free" length. At the tips of the rod free-end boundary conditions were applied and between the sections, correspondingly, the conditions of congruence.

Assuming the hypotheses of Bernoulli-Euler beam theory for the analytical formulation, we chose to neglect the shear deformation and rotational inertia, because the subject of this study was a slender rod, for which the ratio of linear dimensions of cross-sections to length is a very small number.

The energy approach was used to obtain the equations of motion via Lagrangian of the system [1]. Thus, the energy functionals were given by (1)-(4) as follows.

Kinetic energy is

$$
T=\frac{1}{2} \int_{0}^{L}\left[\rho A\left(\frac{\partial w}{\partial t}\right)^{2}+\rho I\left(\frac{\partial^{2} w}{\partial x \partial t}\right)^{2}\right] d x
$$


Potential energy of elastic strain is

$$
U=\frac{1}{2} \int_{0}^{L}\left[E I\left(\frac{\partial^{2} w}{\partial x^{2}}\right)^{2}\right] d x
$$

Potential energy associated with the axial load is

$$
V_{F}=\frac{1}{2} \int_{0}^{L}\left[F\left(\frac{\partial w}{\partial x}\right)^{2}\right] d x
$$

Potential energy associated with the elastic foundation is

$$
V_{w}=\frac{1}{2} \int_{0}^{L} K_{f} w^{2}\left[H\left(l_{f}-x\right)+H\left(x-L+l_{f}\right)\right] d x .
$$

Total potential energy is

$$
\Pi=U+V_{F}+V_{w}
$$

The Heaviside function $H(x)$ in (4) allows us to write down a single expression for the whole tie-rod, taking into account different conditions for its parts.

We proceeded defining the Lagrangian of the system, which is equal to the difference between the overall kinetic and potential energy:

$$
L\left(w, \frac{\partial w}{\partial x}, \frac{\partial w}{\partial t}, \frac{\partial^{2} w}{\partial x^{2}}, \frac{\partial^{2} w}{\partial x \partial t}\right)=T-\Pi
$$

Applying Hamilton's principle, we obtained the system of Lagrange equations of motion (7), where $q_{k}$ indicated every generalized coordinate (degree of freedom) and the number of differential equations was equal to the number of dofs. In our case the degree of freedom was represented by the function $w(x, t)$.

$$
\frac{\partial}{\partial t}\left(\frac{\partial L}{\partial \dot{q}_{k}}\right)-\frac{\partial L}{\partial q_{k}}=0
$$

Sequentially substituting (1)-(5) into (6) and then differentiating the Lagrangian as shown in (7), we obtained the equation of natural vibrations of the rod:

$$
\begin{aligned}
& E I \frac{\partial^{4} w}{\partial x^{4}}+\rho A \frac{\partial^{2} w}{\partial t^{2}}-F \frac{\partial^{2} w}{\partial x^{2}} \\
& \quad+K_{f} w\left[H\left(l_{f}-x\right)+H\left(x-L+l_{f}\right)\right]=0 .
\end{aligned}
$$

As stated above, the ends of tie-rods (where $x=0$ and $x=L$ ) were considered free and between parts I-II and II-III eight conditions of continuity emerged; this provides twelve conditions that were expressed in form of the following:

$$
\begin{aligned}
& x=0:\left\{\begin{array}{l}
Q_{\mathrm{I}}=0 \\
M_{\mathrm{I}}=0,
\end{array}\right. \\
& x=L:\left\{\begin{array}{l}
Q_{\mathrm{III}}=0 \\
M_{\mathrm{III}}=0,
\end{array}\right. \\
& x=l_{f}:\left\{\begin{array}{l}
w_{\mathrm{I}}=w_{\mathrm{II}} \\
\frac{\partial w_{\mathrm{I}}}{\partial x}=-\frac{\partial w_{\mathrm{II}}}{\partial x} \\
\mathrm{Q}_{\mathrm{I}}=Q_{\mathrm{II}} \\
M_{\mathrm{I}}=M_{\mathrm{II}},
\end{array}\right. \\
& x=L-l_{f}:\left\{\begin{array}{l}
w_{\mathrm{II}}=w_{\mathrm{III}} \\
\frac{\partial w_{\mathrm{II}}}{\partial x}=-\frac{\partial w_{\mathrm{III}}}{\partial x} \\
\mathrm{Q}_{\mathrm{II}}=Q_{\mathrm{III}} \\
M_{\mathrm{II}}=M_{\mathrm{III}}
\end{array}\right.
\end{aligned}
$$

The shearing force $Q$ and the bending moment $M$ were defined by

$$
\begin{aligned}
& Q=E I \frac{\partial^{3} w}{\partial x^{3}}-F \frac{\partial w}{\partial x}, \\
& M=E I \frac{\partial^{2} w}{\partial x^{2}} .
\end{aligned}
$$

For each section of the tie-rod (see Figure 2 for the reference system), a separate function $w$ was introduced: $w_{\mathrm{I}}(x, t)$, $w_{\text {II }}(x, t), w_{\text {III }}(x, t)$. The differential equation of motion (8) was solved by means of the Fourier method (11), where $W_{i}(x)$ is the form function, $\tau_{i}(t)$ is the time function, and $i$ takes on a value from 1 to 3 according to each section of the rod.

$$
w_{i}(x, t)=W_{i}(x) \tau_{i}(t)
$$

It is evident that the parts vibrate with the same time frequency; thus, we could get rid of the index $i$ in $\tau_{i}(t)$. For the time function we obtained (12) for all three sections of the rod, with $\omega$ being a natural frequency in $\mathrm{rad} / \mathrm{s}$ and $B_{1}$ and $B_{2}$ being coefficients defining the phase.

$$
\tau(t)=B_{1} \cos \omega t+B_{2} \sin \omega t
$$

After the substitution of (12) and (11) into (8), the form of (13) for parts I, III, and II was delivered.

$$
\frac{d^{4} W_{\mathrm{II}}}{d x^{4}}-\frac{F}{\rho A c^{2}} \frac{d^{2} W_{\mathrm{II}}}{d x^{2}}-\frac{\omega^{2}}{c^{2}} W_{\mathrm{II}}=0,
$$

$$
\frac{d^{4} W_{\mathrm{I}, \mathrm{III}}}{d x^{4}}-\frac{F}{\rho A c^{2}} \frac{d^{2} W_{\mathrm{I}, \mathrm{III}}}{d x^{2}}+\left(\frac{K_{f}}{\rho A c^{2}}-\frac{\omega^{2}}{c^{2}}\right) W_{\mathrm{I}, \mathrm{III}}=0 .
$$


Ordinary differential equations (13) were solved using the general solution given as

$$
W_{\mathrm{I}, \mathrm{II}, \mathrm{III}}(x)=C e^{s x} .
$$

Further, the algebraic equations (15) in coefficients $s$ were obtained throughout substitution of the general solution (14) into the differential equations (13).

$$
\begin{array}{r}
s_{\mathrm{II}}^{4}-\frac{F}{\rho A c^{2}} s_{\mathrm{II}}^{2}-\frac{\omega^{2}}{c^{2}}=0, \\
s_{\mathrm{I}, \mathrm{III}}^{4}-\frac{F}{\rho A c^{2}} s_{\mathrm{I}, \mathrm{III}}^{2}+\left(\frac{K_{f}}{\rho A c^{2}}-\frac{\omega^{2}}{c^{2}}\right)=0 .
\end{array}
$$

Each of equations in (15) provided correspondingly four solutions for $s$ : two complex $s_{3}= \pm i k_{3}$ and two real roots $s_{4}= \pm k_{4}$ for the first equation and four complex roots $s_{1,2}=$ $\pm k_{1} \pm i k_{2}$ for the second one. All of the parameters $s$ in a certain form contain $\omega, F$, and $K_{f}$. Still, to keep our expressions simplified, we used parameters $s$ in the form functions for the natural modes of the tie-rod:

$$
\begin{gathered}
W_{\mathrm{I}}(x)=C_{1} e^{s_{1} x}+C_{2} e^{-s_{1} x}+C_{3} e^{s_{2} x}+C_{4} e^{-s_{2} x}, \\
W_{\mathrm{II}}(x)=C_{5} e^{s_{3} x}+C_{6} e^{-s_{3} x}+C_{7} e^{s_{4} x}+C_{8} e^{-s_{4} x}, \\
W_{\mathrm{III}}(x)=C_{9} e^{s_{1} x}+C_{10} e^{-s_{1} x}+C_{11} e^{s_{2} x}+C_{12} e^{-s_{2} x} .
\end{gathered}
$$

Substituting the forms (16) into twelve conditions (9), we hence obtained a homogeneous system of twelve equations containing twelve unknowns $C_{1} \cdots C_{12}$, which in a matrix form is expressed as

$$
[M]\{C\}=\{0\} .
$$

In order to provide a nontrivial solution the determinant of the matrix $M$ in (17) was supposed to be equal to zero (18), which resulted in a characteristic equation of the eigenvalue problem.

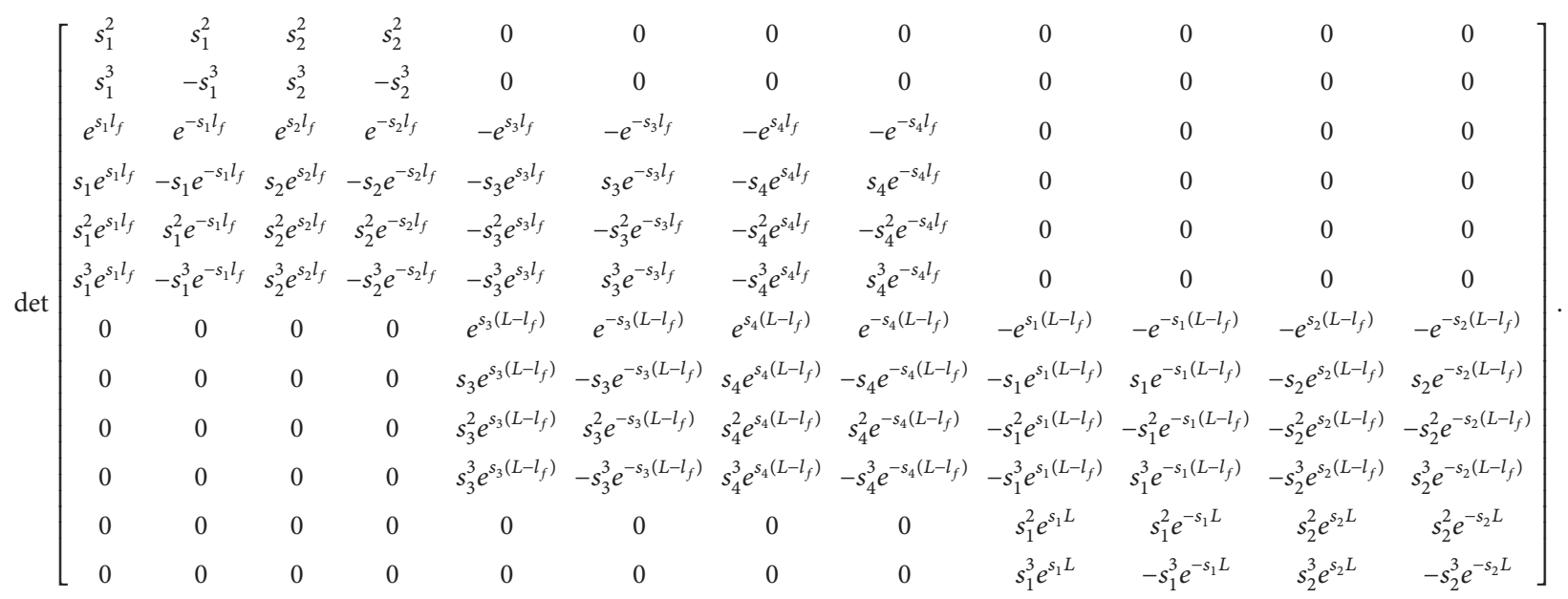

Equation (18) is to be solved for the natural frequencies $\omega$ by means of, for instance, the Newton-Raphson method. Furthermore, the dispersion between analytically and experimentally determined frequencies can be minimized with respect to parameters $F$ and $K_{f}$, following the optimization procedure described in the next sections. This approach in a closed form delivers solution for the sought axial load in tierod.

\subsection{Numerical Model and Optimization Procedure. Tie-rods} were modelled in FEM software using beam elements. In this case the beam is represented with a one-dimension body, that is, a wire (line, curve, polyline), and cross-section shapes and dimensions are assigned to this body as one of the properties, which allowed taking into account irregular cross-sections, added masses, elastic supports, and so forth. The beam model shows good results for analyses of long slender beams. For beam elements there is an option of modelling the axial tensile load as a bolt pretension load. Tie-rods were modelled using 50-60 B31 2-node linear beam elements in 3D space implying Timoshenko's beam theory.

The FEM simulation was divided into two steps: as a first step a pretension load $F$ was applied to the beam and as a second step the modal analysis was performed. The FEM model was parametric, since the tensile load and elastic foundation parameters were unknown. The idea here was to "tune" these parameters in order to match results of physical tests and FEM simulations. Optimization criterion given by (19) represented a residual error between $n$ natural frequencies defined via experimental modal analysis $\left\{f_{1}^{\exp }, \ldots, f_{n}^{\exp }\right\}$ and numerically $\left\{f_{1}^{\mathrm{FEM}}, \ldots, f_{n}^{\mathrm{FEM}}\right\}$. The error function contained weight coefficients $p_{k}$ arbitrary assigned to each natural mode. Hence, the minimum of the multiparameter function $R$ delivered the optimal solution.

$$
R=\sqrt{\sum_{k=1}^{n} p_{k}^{2}\left(f_{k}^{\exp }-f_{k}^{\mathrm{FEM}}\right)^{2}} .
$$



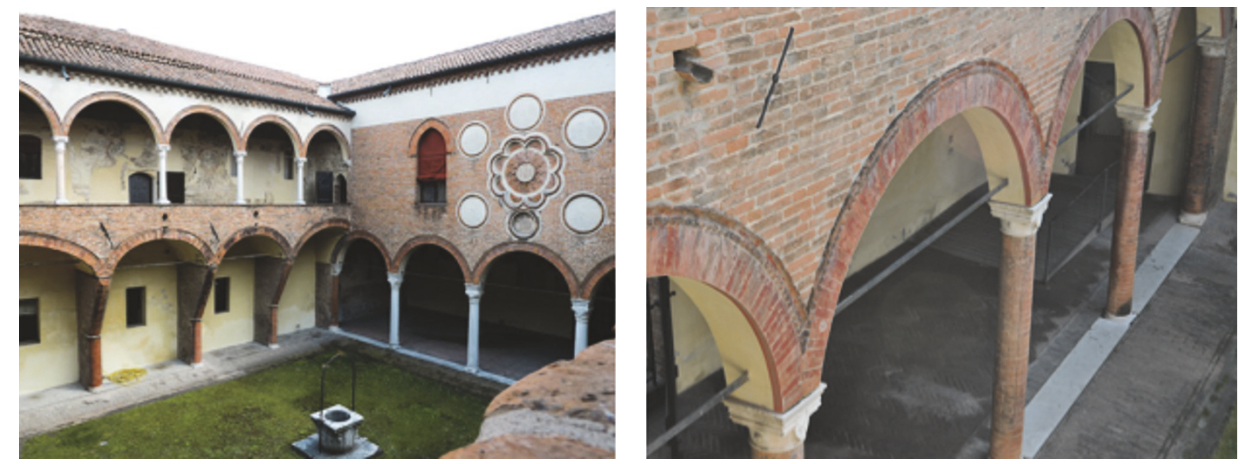

FIGURE 3: Inner yard of Casa Romei and zoom of the ground floor tie-rods.
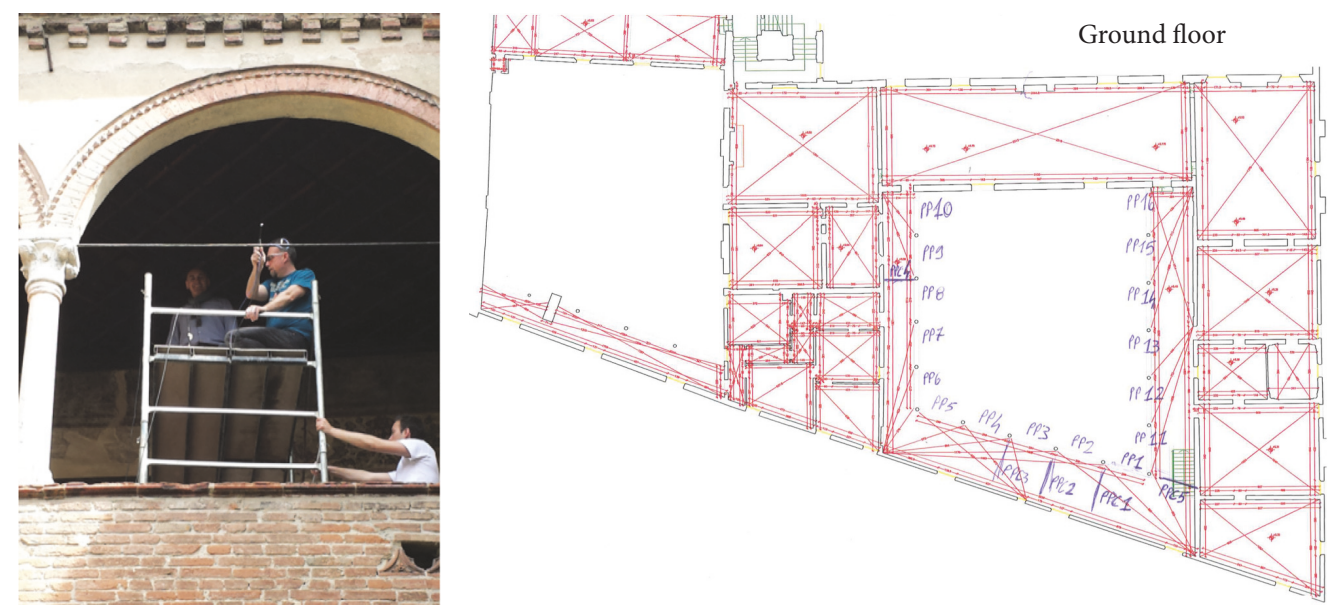

FIGURE 4: Our team during hammer excitation of a tie-rod; map of ground floor.

By using a parametric FEM model the representation of a tie-rod became flexible and first 6 natural frequencies were matched with overall accuracy up to $1 \%$. Parameterisation of the FEM model was achieved by direct coding of the analysis input file, since parametric analysis is not enabled inside the graphical interface of Abaqus. Afterwards the script was executed automatically inside the optimization program coded in C. The latter program automatically extracted the required results from the text files with analysis outputs created by Abaqus.

As a first iteration we analytically investigated the function $R$ (19) for a tie-rod with fixed and/or simply supported conditions, with length $L$ and axial load $F$ being optimization parameters. On a reasonable range of optimization parameters the residual error (19) had only a forced minimum at the minimal value of the length parameter. The found absolute minimum was lying, however, below the measured length of a tie-rod. This behaviour proved the necessity of modelling more complex boundary conditions. However, this first iteration provided a rough idea of the sought axial load and we could set the range for $F$ around this value.

Subsequently we modelled the elastic bed boundaries, representing a general condition of translational and rotational stiffness acting for the length $l_{f}$, as in the sketch displayed in Figure 2. Elastic bed consists of equispaced linear elastic springs each of stiffness $k_{f}$. Further the optimization has been done with respect to the sought axial load and the parameters of elastic foundation. We needed to provide sets of experimentally obtained frequencies, ranges, and step sizes of optimization parameters and sets of weight coefficients $p_{k}$ to the code in $\mathrm{C}$ that

(i) forms a matrix of parameters;

(ii) launches the FEM analysis for each nod of the grid, extracts and filters natural frequencies from the output;

(iii) calculates the value of residual error (19) for each step;

(iv) finds the local minimum of the function (19) and the corresponding combination of parameters including the sought tensile load;

(v) refines the grid of parameters and repeats the procedure again.

3.3. Application to a Case Study. The method described hereby was applied to investigation of tie-rods installed in "Casa Romei" located in Ferrara, Italy (see Figures 3 and 4). Romeì House is a perfect example of a 15th century palace, in which you can see rich gothic decoration of the Late Middle 
TABLE 1: Experimental acquisitions.

\begin{tabular}{|c|c|c|c|c|c|c|c|c|}
\hline \multirow{2}{*}{ Tie-rod number } & \multirow{2}{*}{ Cross-section $A\left[\mathrm{~mm}^{2}\right]$} & \multirow{2}{*}{ Length $l[\mathrm{~mm}]$} & \multicolumn{6}{|c|}{ Natural frequencies $[\mathrm{Hz}]$} \\
\hline & & & I & II & III & IV & $\mathrm{V}$ & VI \\
\hline PT1 & $52 \times 9$ & 3178 & 15.30 & - & 49.00 & - & 92.30 & - \\
\hline PT2 & $51 \times 9$ & 3233 & 16.80 & - & 53.80 & - & 98.80 & - \\
\hline PT3 & $52 \times 10$ & 3228 & 16.30 & - & 53.80 & - & 103.00 & - \\
\hline PT4 & $51 \times 10$ & 3218 & 16.00 & 33.50 & 51.30 & 71.80 & 95.00 & 121.80 \\
\hline PT5 & $53 \times 10$ & 3188 & 5.50 & - & 28.30 & - & 65.50 & 91.00 \\
\hline PT6 & $50 \times 20$ & 2748 & 21.75 & 49.00 & 85.25 & 131.20 & 188.50 & 255.20 \\
\hline PT7 & $50 \times 20$ & 2768 & 20.00 & 45.50 & 80.00 & 123.80 & 179.20 & 242.20 \\
\hline PT8 & $50 \times 20$ & 3358 & 14.75 & 32.75 & 55.75 & 85.25 & 121.20 & 176.50 \\
\hline PT9 & $50 \times 20$ & 3248 & 15.50 & 34.25 & 58.75 & 90.00 & 128.00 & 173.00 \\
\hline PT10 & $50 \times 20$ & 3388 & 17.75 & 38.00 & 63.25 & 94.75 & 133.00 & 177.50 \\
\hline PT11 & $50 \times 12$ & 3440 & 13.25 & 29.75 & 51.25 & 78.25 & 111.50 & 150.80 \\
\hline PT12 & $50 \times 12$ & 3298 & 14.50 & 30.25 & 48.25 & 69.25 & 94.00 & 122.20 \\
\hline PT13 & $50 \times 12$ & 3140 & 13.75 & - & 47.25 & - & 95.75 & - \\
\hline PT14 & $50 \times 12$ & 2510 & 19.25 & 46.50 & & 84.00 & - & 134.50 \\
\hline
\end{tabular}

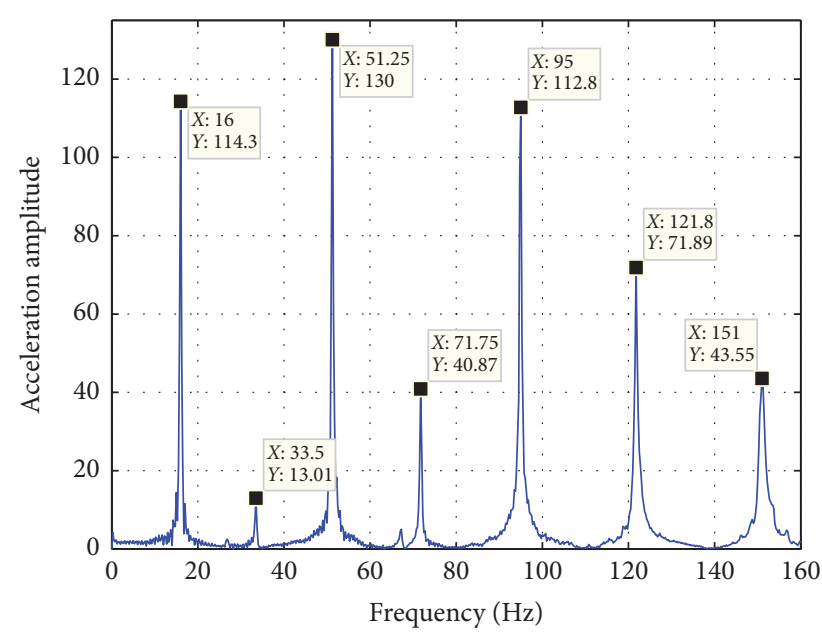

FIGURE 5: FRF plot (acceleration amplitude versus frequency).

Ages combine with elements of the Early Renaissance. Tierods have been placed in this building in different times along its existence, differing in dimensions and cross-section shapes.

First, measurements of geometrical characteristics and of natural frequencies were performed for each tie-rod. Then experimental acquisition was carried out to determine the natural frequencies from the analysis of response to dynamical excitation applied to tie-rods in horizontal plane; as an example, a frequency response function (FRF) for a ground floor tie-rod is shown in Figure 5.

For further analysis, first four to six natural frequencies were identified for each tie-rod. Six eigenmodes were considered sufficient, since identification of higher modes might appear inaccurate due to larger possible measurement errors.

Having performed multiple experimental studies of tierods, the authors concluded that the variation of the material was less significant than in the boundary conditions. Thus,

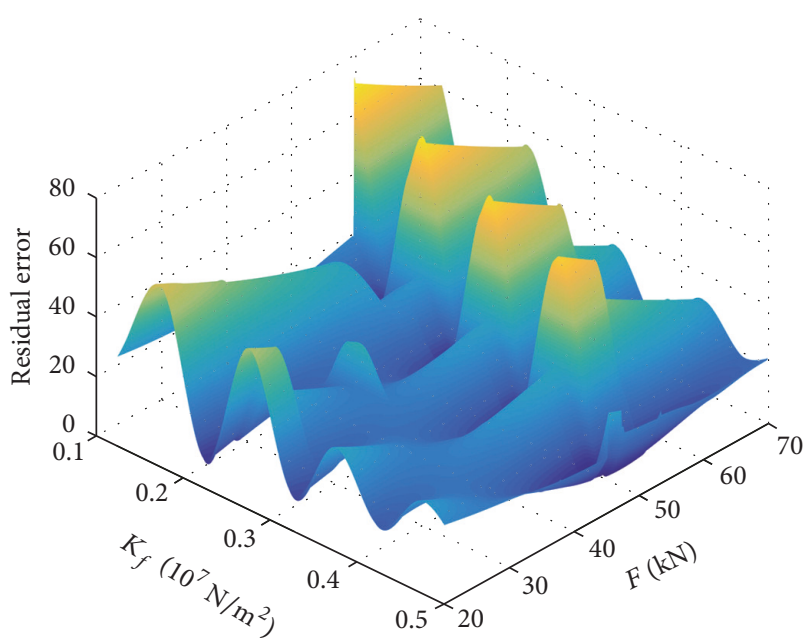

FIGURE 6: Residual error function as function of elastic bed stiffness and axial load.

the material properties were kept constant in this case study: material was assumed to be general iron with characteristics: $E=210 \mathrm{GPa}, \nu=0.3, \rho=7850 \mathrm{~kg} / \mathrm{m}^{3}$.

3.4. Load Identification Results. Table 1 summarizes the data on cross-section $A$, free length $l$, and natural frequencies of the ground floor tie-rods (see Figure 4 for layout). Some of the frequencies in Table 1 are missing, due to the eventual position of the accelerometer over a modal node; in these cases higher frequencies were considered in the optimization process.

A typical graphic scenario of the optimization process is depicted in Figure 6. Here several values of the function $R$ (19) were plotted versus axial load $F$ and distributed stiffness of the elastic bed $K_{f}$, for the tie-rod PT2. The length of elastic bed $l_{f}$ (see Figure 2) was kept constant. It is notable that many minima are present in correspondence to certain $F$ $K_{f}$ couples and one of them is a local minimum. The grid 
TABLE 2: Summary of results.

\begin{tabular}{|c|c|c|c|c|}
\hline Tie-rod number & Axial load $F[\mathrm{kN}]$ & Stress [MPa] & Bed stiffness $K_{f}\left[10^{7} \mathrm{MN} / \mathrm{m}^{2}\right]$ & Residual error \\
\hline PT1 & 29.40 & 62.82 & 50.00 & 0.31 \\
\hline PT2 & 46.90 & 102.18 & 0.48 & 6.04 \\
\hline PT3 & 37.50 & 72.12 & 253.00 & 3.95 \\
\hline PT4 & 38.70 & 75.88 & 3.75 & 0.77 \\
\hline PT5 & 1.00 & 1.89 & 26.75 & 1.17 \\
\hline PT6 & 66.50 & 66.50 & 113.00 & 0.48 \\
\hline PT7 & 54.50 & 54.50 & 57.50 & 1.43 \\
\hline PT8 & 46.30 & 46.30 & 60.00 & 6.60 \\
\hline PT9 & 44.50 & 44.50 & 62.50 & 7.33 \\
\hline PT10 & 79.90 & 79.90 & 76.00 & 2.22 \\
\hline PT11 & 30.10 & 50.17 & 99.50 & 6.53 \\
\hline PT12 & 37.20 & 62.00 & 4.25 & 0.48 \\
\hline PT13 & 28.20 & 47.00 & 5.50 & 0.27 \\
\hline PT14 & 38.00 & 63.33 & 2.13 & 5.58 \\
\hline
\end{tabular}

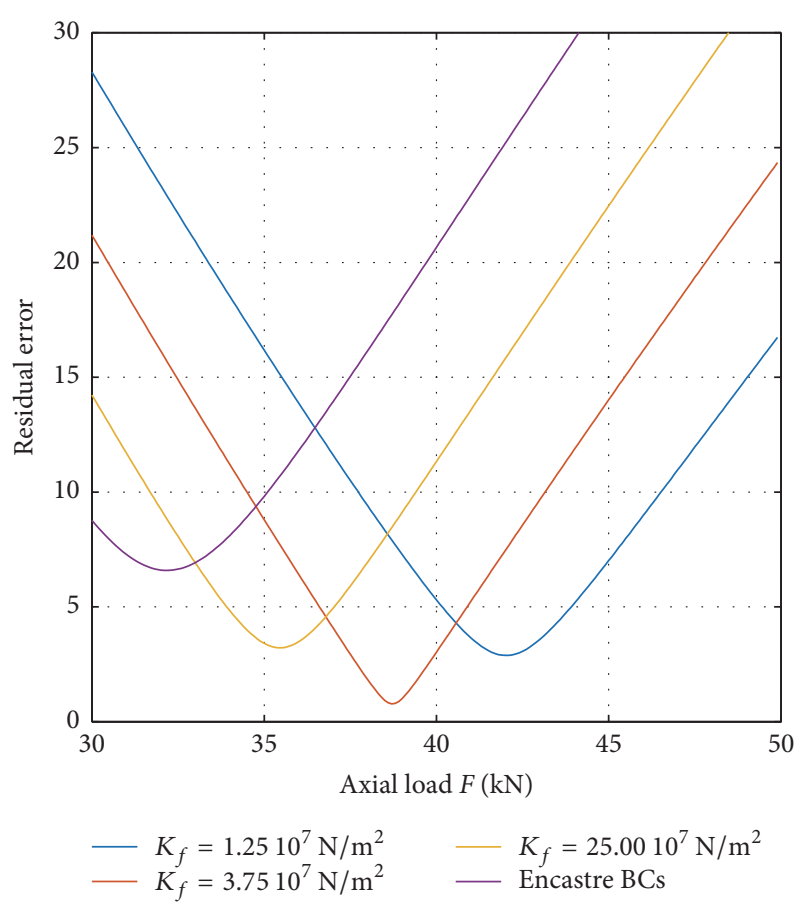

FIGURE 7: Influence of the elastic bed on the axial load determination.

of parameters $F$ and $K_{f}$ was then reduced around it, in order to refine the search of the optimal values.

Naturally, the so-defined minima of the residual error (19) were not independent; nevertheless, once the main parameter (axial load $F$ ) was optimized, the influence of stiffness of constraints on the first parameter was nearly negligible.

The model with elastic foundation boundaries delivered improvement of results as illustrated in Figure 7 . The plot shows residual error curves for the tie-rod PT4 at different values of stiffness $K_{f}$ compared to the clamped boundaries. It indicates that the minimum of the error (19) decreases from 6.59 (encastré BCs) to 0.77 for the optimal stiffness $K_{f}=$
$3.75 \times 10^{7} \mathrm{~N} / \mathrm{m}^{2}$. The corresponding optimal value of the axial load in this tie-rod increases by $20 \%$ from $32.20 \mathrm{kN}$ (encastré $\mathrm{BCs}$ ) to $38.70 \mathrm{kN}$ (elastic bed). We presume that a model with simple clamped boundaries underestimated the axial load; thus, the proposed method reveals to be conservative for the load estimation.

Table 2 summarizes the results of the computation process of the reported case study.

3.5. Analysis of the Results. Tensile loads cause normal stresses that need to be estimated for evaluation of reliability and integrity of tie-rods. This safety assessment can be carried out based on the values of average axial stress $\bar{\sigma}_{k, N}$, to which each tie-rod is subjected. The stress was calculated with respect to the optimal axial load $F_{k, \text { opt }}$ and the minimum cross-section area $A_{k \text {, min }}$ :

$$
\bar{\sigma}_{k, N}=\frac{F_{k, \mathrm{opt}}}{A_{k, \mathrm{~min}}} .
$$

This stress (20) is average since it assumes a uniform section without taking into consideration such local effects as screws, fillets, holes, joints, and so forth that tie-rods might incorporate. However, these stress concentrators should be considered, if present, for correct local strength verification, eventually via FEM analysis.

In Figure 8 the stresses in rods PT1-PT14 are plotted. Apart from PT5, all tie-rods worked properly and below the allowable stress of $120 \mathrm{MPa}$ for ancient iron. Rod PT5 was evidently unloaded, which indicated that there was either some damage or malfunction at the anchorages; the issue has been stated in the appropriate technical report of the case study. For comparison, Figure 8 reports also average stresses calculated for the case of encastré boundary conditions: safety improvement was definitely confirmed.

In Section 3.2 we introduced the residual error (19) to compute "distance" between experimental and numerical frequencies. In the formula the difference (in $\mathrm{Hz}$ ) between each couple of frequencies is multiplied by a weight coefficient 


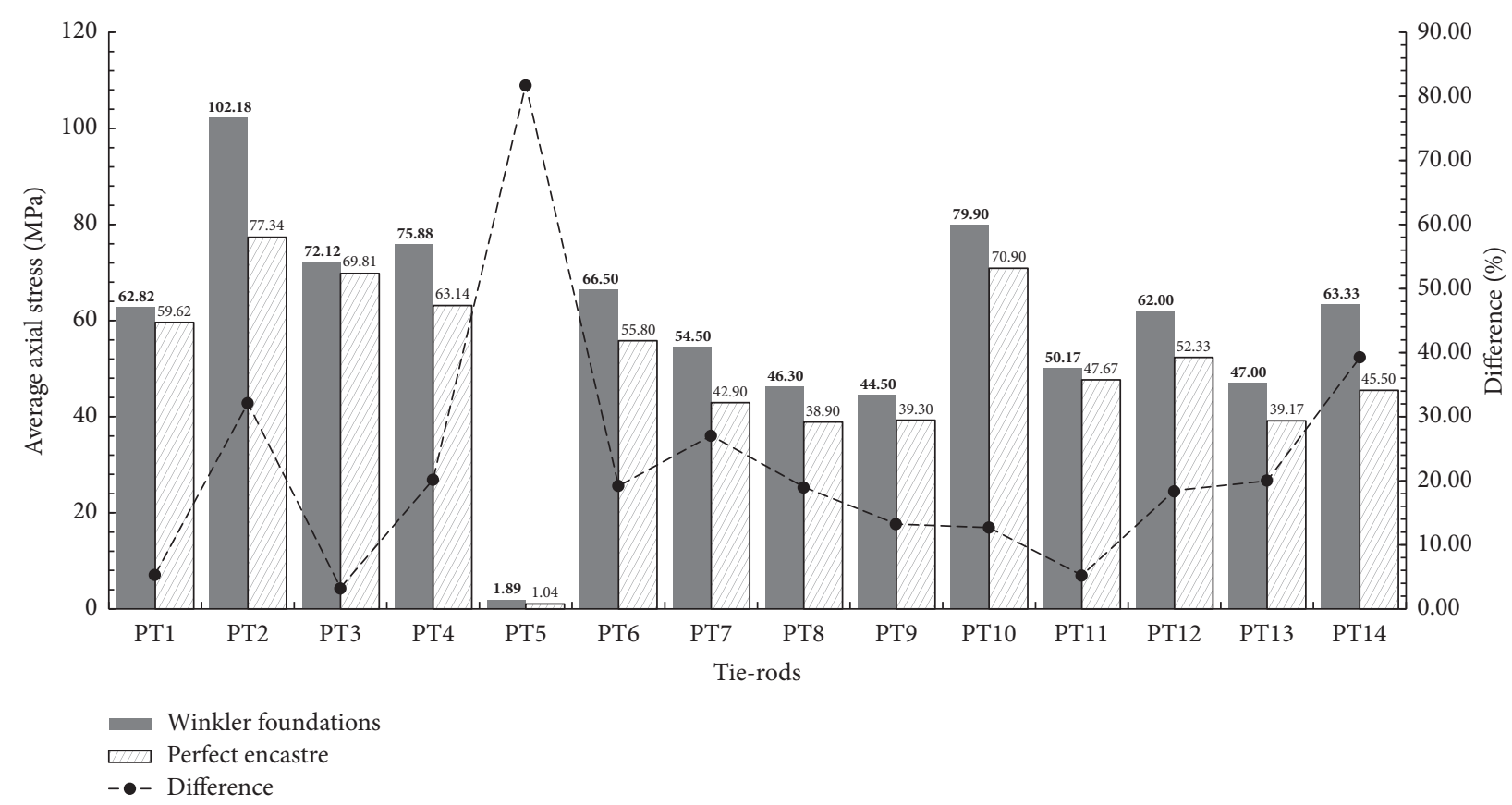

FIGURE 8: Average stress state in tie-rods: comparison between encastré and elastic foundation boundaries.

TABLE 3: Results of computation with three sets of weights for the tie-rod PT4.

\begin{tabular}{|c|c|c|c|c|c|c|c|c|}
\hline \multirow{2}{*}{ Sets of weight coefficients } & \multirow{2}{*}{ Axial load $F[\mathrm{kN}]$} & \multirow{2}{*}{ Bed stiffness $K_{f}\left[10^{7} \mathrm{MN} / \mathrm{m}^{2}\right]$} & \multicolumn{6}{|c|}{ Natural frequencies $[\mathrm{Hz}]$} \\
\hline & & & I & II & III & IV & $\mathrm{V}$ & VI \\
\hline$W_{1}=\{1,1,1,1,1,1\}$ & 39.00 & 3.50 & 16.03 & 32.91 & 51.37 & 72.03 & 95.32 & 121.53 \\
\hline$W_{3}=\{4,1,0.5,0.25,0.1,0.05\}$ & 38.60 & 4.50 & 16.04 & 32.95 & 51.48 & 72.25 & 95.69 & 122.13 \\
\hline$W_{2}=\{10,1,1,1,1,1\}$ optimal values & 38.70 & 3.75 & 16.00 & 32.85 & 51.31 & 71.97 & 95.28 & 121.55 \\
\hline$W_{2}$, encastré $\mathrm{BCs}$ & 32.20 & $\infty$ & 15.68 & 32.47 & 51.33 & 72.97 & 97.91 & 126.50 \\
\hline Experimentally defined frequencies & & & 16.00 & 33.50 & 51.30 & 71.80 & 95.00 & 121.80 \\
\hline
\end{tabular}

$p_{k}$ in order to attribute higher or lower importance to some frequencies rather than others. The set of weight coefficients was arbitrary chosen, but generally higher importance has been given to the first frequencies.

A sensitivity analysis has been conducted to evaluate the influence of weight coefficients on our results. The plot in Figure 9 shows three residual error functions for the tie-rod PT4 corresponding to three different sets of weight coefficients. The sets taken into consideration were $W_{1}=\{1,1,1,1,1,1\}, W_{2}=\{10,1,1,1,1,1\}$, and $W_{3}=$ $\{4,1,0.5,0.25,0.1,0.05\}$. We see that each set generated a different interpolated surface; however, the error reached minimum around the same value of the sought axial load, as reported in Table 3. Many other reasonable sets of weight coefficients have been tested, confirming their low influence on the resulting axial load, which proved the stability of our model with respect to these arbitrary assigned parameters.

\section{Conclusions}

In this paper a procedure for axial load identification in structural tie-rods was demonstrated and approved via an experimental study of an ancient mansion. The method is

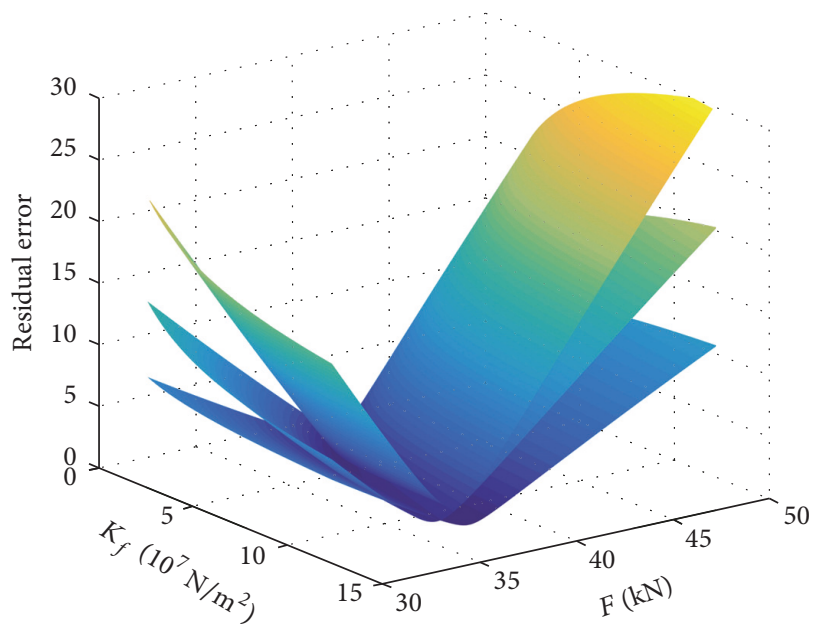

FIGURE 9: Residual error functions for sets of weight coefficients $W_{1}$, $W_{2}, W_{3}$.

based on a tie-rod model represented by a beam with ends supported by an elastic Winkler-type foundation. The elastic bed was used to simulate the contact condition between a 
tie-rod and a masonry wall. The proposed method consisted of an experimental and a computational stage. The experimental part was a relatively simple vibrational test for natural frequencies identification. The computational part was an optimization procedure for axial load estimation based on finite element modelling. The optimization has been done with respect to two parameters: the sought axial load and the distributed stiffness of the elastic bed at the boundaries. The technique provided a solution for uncertain boundary conditions and is capable of identifying axial load with high accuracy.

Investigation of the behaviour of natural frequencies depending on the parameters showed that axial load tends to shift the set of frequencies (the higher the load the higher the frequencies), while the elastic foundation stiffness changes the "distance" between natural frequencies.

As a result, consideration of elasticity at anchorages exhibited increase in axial load by up to $40 \%$. This means that assumption of simple boundary conditions is not sufficient for modelling a tie-rod dynamic response. The sensitivity analysis has proved that the optimization result was stable to variation of weight coefficients and was converging to the same axial load. Thus, the method has been approved in practice and is suitable for in situ identification of axial load in ancient tie-rods.

\section{Nomenclature}

$A$ : $\quad$ Cross-section area of the tie-rod

$B_{1}, B_{2}$ : Coefficients in the time function of the solution for tie-rod deflection

C: $\quad$ Coefficient of the general solution for the form function

$C_{1}, \ldots, C_{12}$ : Coefficients of the solution for tie-rod deflection that satisfy the specific boundary conditions

C: $\quad$ Constant equal to the ratio $\sqrt{E I / \rho A}$

$E: \quad$ Elastic modulus of the tie-rod material

F: $\quad$ Axial load acting on the tie-rod

$f_{k}^{\text {exp }}$ : $\quad$ Natural frequency number $k$ determined experimentally

$f_{k}^{\mathrm{FEM}}$ : $\quad$ Natural frequency number $k$ determined from FEM

$H(x)$ : Heaviside function

I: $\quad$ Moment of inertia of the cross-section about $z$-axis

$K_{f}: \quad$ Distributed stiffness of the elastic bed

$k_{f}$ : $\quad$ Stiffness of the separate springs that discretize the elastic bed

$k_{1}, \ldots, k_{4}$ : Real constants used in the expressions for $s$

$L: \quad$ Total length of the tie-rod

$l_{f}$ : $\quad$ Lengths of the tie-rod portion inserted into the wall, sections I and III

$L: \quad \quad$ "Free" length of the tie-rod, section II

M: $\quad$ Matrix of the system of linear algebraic equations for the unknowns $C_{1}, \ldots, C_{12}$

$N$ : Poisson's ratio

\begin{tabular}{|c|c|}
\hline$\Pi:$ & Total potential energy \\
\hline$p_{k}:$ & $\begin{array}{l}\text { Weight coefficient of a frequency } \\
\text { number } k\end{array}$ \\
\hline$R:$ & $\begin{array}{l}\text { Residual error between two sets of } n \\
\text { natural frequencies }\end{array}$ \\
\hline$P:$ & Density of the tie-rod material \\
\hline$s, s_{\mathrm{I}, \mathrm{II}, \mathrm{III}}, s_{1}, \ldots, s_{4}$ & $\begin{array}{l}\text { Coefficients in the exponent of the } \\
\text { solution for tie-rod deflection }\end{array}$ \\
\hline$T:$ & Kinetic energy \\
\hline$\tau_{i}(t), \tau(t):$ & $\begin{array}{l}\text { Time function in the Fourier solution } \\
\text { for tie-rod deflection }\end{array}$ \\
\hline$U:$ & Potential energy of elastic strain \\
\hline$V_{F}:$ & $\begin{array}{l}\text { Potential energy associated with the } \\
\text { axial load }\end{array}$ \\
\hline$V_{w}:$ & $\begin{array}{l}\text { Potential energy associated with the } \\
\text { elastic foundation }\end{array}$ \\
\hline$\Omega:$ & Natural frequency in $\mathrm{rad} / \mathrm{s}$ \\
\hline$w(x, t):$ & Tie-rod deflection in $x O y$ plane \\
\hline$w_{\text {IJIUI }}(x, t):$ & Deflection for each section of the tie-rod \\
\hline$W(x):$ & Form function of the tie-rod deflection \\
\hline$W_{\mathrm{I}, \mathrm{II}, \mathrm{III}}(x):$ & $\begin{array}{l}\text { Form functions for each section of the } \\
\text { tie-rod. }\end{array}$ \\
\hline
\end{tabular}

\section{Conflicts of Interest}

The authors declare that there are no conflicts of interest regarding the publication of this paper.

\section{References}

[1] M. Amabili, S. Carra, L. Collini, R. Garziera, and A. Panno, "Estimation of tensile force in tie-rods using a frequency-based identification method," Journal of Sound and Vibration, vol. 329, no. 11, pp. 2057-2067, 2010.

[2] R. Garziera, M. Amabili, and L. Collini, "A hybrid method for the nondestructive evaluation of the axial load in structural tierods," Nondestructive Testing and Evaluation, vol. 26, no. 2, pp. 197-208, 2011.

[3] L. Collini, R. Fagiani, R. Garziera, K. Riabova, and M. Vanali, "Load and effectiveness of the tie-rods of an ancient dome: technical and historical aspects," Journal of Cultural Heritage, vol. 16, no. 4, pp. 597-601, 2015.

[4] L. Collini and R. Garziera, "Measurement of the tensile force in the hoop tie-rods of an historical dome," in Proceedings of PROHITECH14 - 2nd International Conference on Protection of Historical Constructions, SYKORA, Antalya, Turkey, 2014.

[5] K. Riabova, L. Collini, and R. Garziera, "Numerical method for the estimation of tensile load in tie-rods", in Proceedings of the 14th Youth Symposium on Experimental Solid Mechanics, vol. 3, Traunkirchen Monastery, Austria, 2015.

[6] L. Collini, R. Garziera, and K. Riabova, "Experimental and numerical determination of the axial load in structural tierods with complex boundary conditions," in Proceedings of the XXXVI Iberian Latin American Congress on Computational Methods in Engineering, SWGE Sistemas, Rio de Janeiro, Brazil, November 2015.

[7] M. Amabili and R. Garziera, "A technique for the systematic choice of admissible functions in the Rayleigh-Ritz method," Journal of Sound and Vibration, vol. 224, no. 3, pp. 519-539, 1999. 
[8] P. Pozzati, Teoria e Tecnica delle Strutture (Theory and Technique of Structures), UTET, Turin, Italy, 1977.

[9] M. L. Beconcini, "Un metodo pratico per la determinazione del tiro nelle catene (a practical method for the determination of tensile load in tie-rods," Costruire in Laterizio 54/96, pp. 299301, 1996.

[10] S. B. Bati and U. Tonietti, "Experimental methods for estimating in situ tensile force intie-rods," Journal of Engineering Mechanics, vol. 127, no. 12, pp. 1275-1283, 2001.

[11] C. Blasi and S. Sorace, Sulla determinazione del "tiro" nelle catene mediante prove statiche e dinamiche, Atti III Congresso Nazionale ASS.I.R.CO, Catania, Italy, November 1988.

[12] C. Blasi and S. Sorace, "Determining the axial force in metallic rods," Structural Engineering International (IABSE), vol. 4, no. 4, pp. 241-246, 1994.

[13] S. Sorace, "Parameter models for estimating in-situ tensile force in tie-rods," Journal of Engineering Mechanics, vol. 122, no. 9, pp. 818-825, 1996.

[14] S. Lagomarsino and C. Calderini, "The dynamical identification of the tensile force in ancient tie-rods," Engineering Structures, vol. 27, no. 6, pp. 846-856, 2005.

[15] K. Maes, J. Peeters, E. Reynders, G. Lombaert, and G. De Roeck, "Identification of axial forces in beam members by local vibration measurements," Journal of Sound and Vibration, vol. 332, no. 21, pp. 5417-5432, 2013.

[16] S. Li, E. Reynders, K. Maes, and G. De Roeck, "Vibration-based estimation of axial force for a beam member with uncertain boundary conditions," Journal of Sound and Vibration, vol. 332, no. 4, pp. 795-806, 2013.

[17] G. Rebecchi, N. Tullini, and F. Laudiero, "Estimate of the axial force in slender beams with unknown boundary conditions using one flexural mode shape," Journal of Sound and Vibration, vol. 332, no. 18, pp. 4122-4135, 2013.

[18] N. Tullini, "Bending tests to estimate the axial force in slender beams with unknown boundary conditions," Mechanics Research Communications, vol. 53, pp. 15-23, 2013.

[19] N. Tullini, G. Rebecchi, and F. Laudiero, "Bending tests to estimate the axial force in tie-rods," Mechanics Research Communications, vol. 44, pp. 57-64, 2012.

[20] N. Tullini and F. Laudiero, "Dynamic identification of beam axial loads using one flexural mode shape," Journal of Sound and Vibration, vol. 318, no. 1-2, pp. 131-147, 2008.

[21] C. Gentilini, A. Marzani, and M. Mazzotti, "Nondestructive characterization of tie-rods by means of dynamic testing, added masses and genetic algorithms," Journal of Sound and Vibration, vol. 332, no. 1, pp. 76-101, 2013.

[22] T. Livingston, J. G. Béliveau, and D. R. Huston, "Estimation of axial load in prismatic members using flexural vibrations," Journal of Sound and Vibration, vol. 179, no. 5, pp. 899-908, 1995.

[23] B. H. Kim and T. Park, "Estimation of cable tension force using the frequency-based system identification method," Journal of Sound and Vibration, vol. 304, no. 3-5, pp. 660-676, 2007.

[24] C. Urbano, "Sulla determinazione del tiro nelle catene degli archi: considerazioni teoriche ed applicazioni sperimentali (on the determination of tensile load in tie-rods of arcs: theoretical considerations and experimental applications)," Costruzioni Metalliche, vol. 5, pp. 315-323, 1967.

[25] M. Arduini, R. Carli, and P. Cesari, “Tecniche di identificazione dello sforzo e del grado di vincolo nei tiranti strutturali con metodi dinamici (identification techniques of stress and the degree of constraint in the structural tie-rods with dynamic methods)," Ingegneri Architetti Costruttori (INARCOS), vol. 563, pp. 493-496, 1995.

[26] E. Viola, M. Dilena, and E. Artioli, "Identification of metallic rods by frequency estimation on the historical church tower in S.Vito al Tagliamento (PN)-Italy," in Proceedings of the International Conference on Restoration, Recycling and Rejuvenation Technology for Engineering and Architecture Application, pp. 173-179, Cesena, Italy, June 2004.

[27] W.-X. Ren, G. Chen, and W.-H. Hu, "Empirical formulas to estimate cable tension by cable fundamental frequency," Structural Engineering and Mechanics, vol. 20, no. 3, pp. 363380, 2005.

[28] S. Park, S. Choi, S.-T. Oh, N. Stubbs, and H.-C. Song, "Identification of the tensile force in high-tension bars using modal sensitivities,' International Journal of Solids and Structures, vol. 43, no. 10, pp. 3185-3196, 2006.

[29] S. H. Farghaly and K. M. Zeid, "An exact frequency equation for an axially loaded beam-mass-spring system resting on a Winkler elastic foundation," Journal of Sound and Vibration, vol. 185, no. 2, pp. 357-363, 1995.

[30] P. Ruge and C. Birk, "A comparison of infinite Timoshenko and Euler-Bernoulli beam models on Winkler foundation in the frequency- and time-domain," Journal of Sound and Vibration, vol. 304, no. 3-5, pp. 932-947, 2007.

[31] S. G. Koroma, M. F. M. Hussein, and J. S. Owen, "Vibration of a beam on continuous elastic foundation with nonhomogeneous stiffness and damping under a harmonically excited mass," Journal of Sound and Vibration, vol. 333, no. 9, pp. 2571-2587, 2014. 


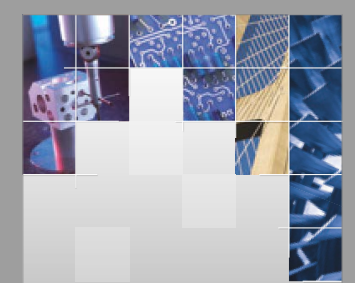

\section{Enfincering}
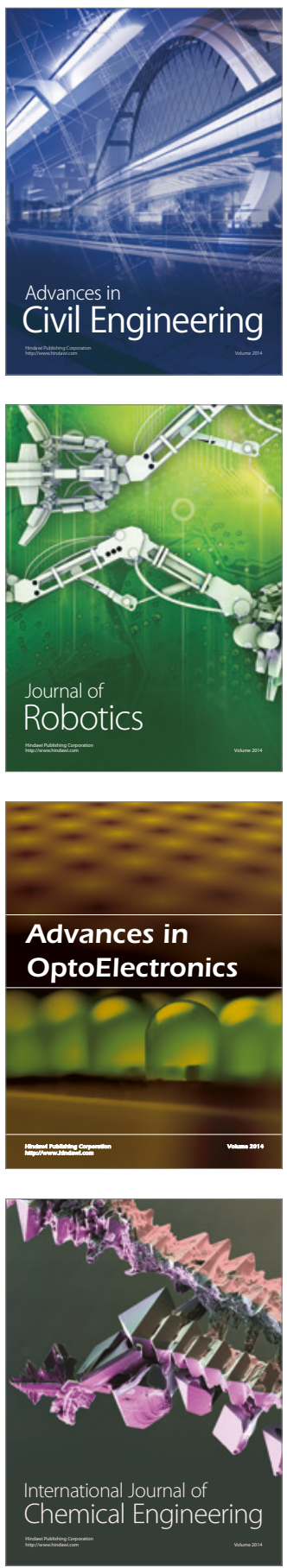

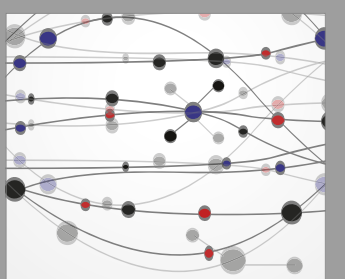

The Scientific World Journal

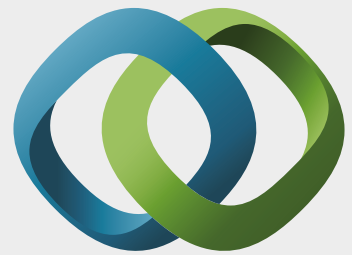

\section{Hindawi}

Submit your manuscripts at

https://www.hindawi.com
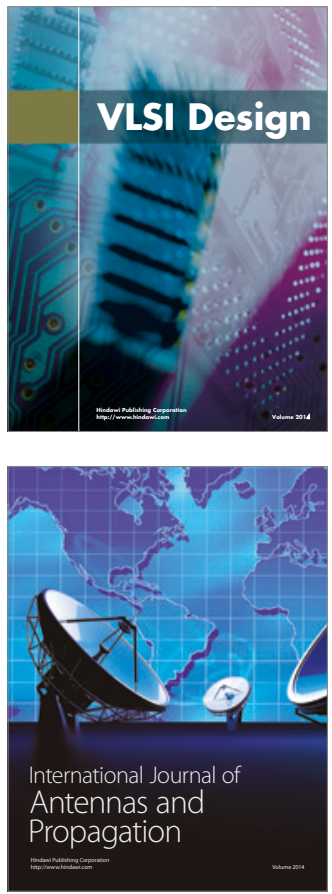

\section{Rotating}

Machinery
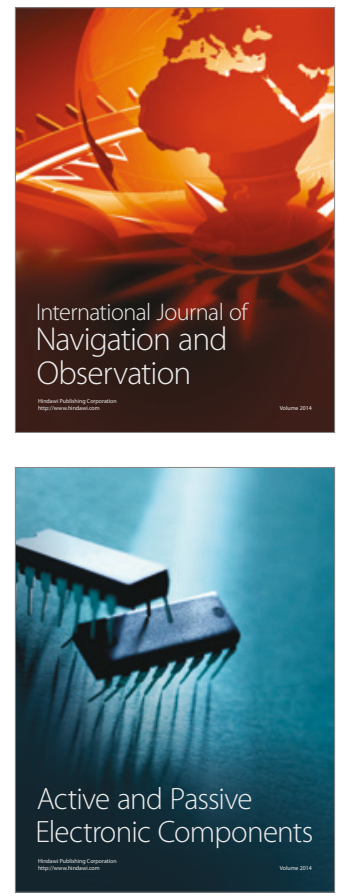
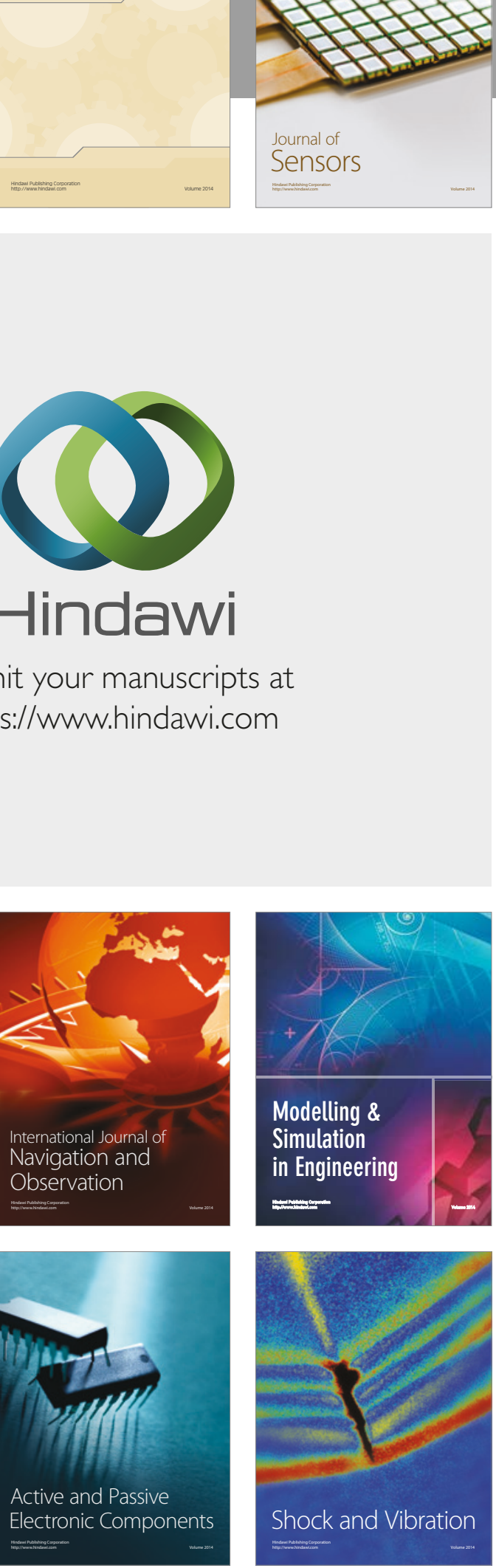
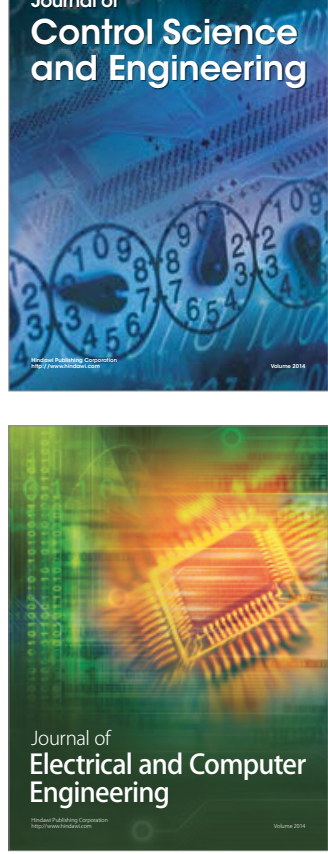

Distributed

Journal of

Control Science

and Engineering
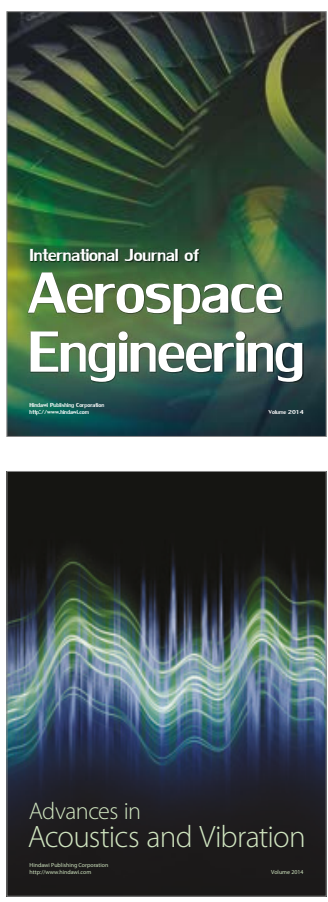

Sensor Networks 